Apidologie, 1986, 17 (2), 79-92

\title{
ÉTUDE BIOMÉTRIQUE DES POPULATIONS D'ABEILLES (APIS MELLIFERA) DU NORD-OUEST DE L'ESPAGNE
}

\author{
E. SANTIAGO, J. ALBORNOZ, A. DOMÍNGUEZ et J.I. IZQUIERDO \\ Departamento Interfacultativo de Genética \\ Facultades de Biologia y Medicina. C/ Julián Clavería sn. 33006 Oviedo. España
}

\begin{abstract}
RÉSUME
On a réalisé une analyse canonique, prenant comme base la mesure de l'aile des ouvrières, sur des spécimens recueillis dans le Nord-Ouest de l'Espagne. Cette région est divisée par la Cordillère Cantabrique en deux zones différentes du point de vue du climat et de la flore. La Cordillère semble être l'élément principal qui établit la différence entre les groupes, bien que l'absence de discontinuité entre ceux-ci suggère qu'elle ne produit pas d'isolement géographique.
\end{abstract}

\section{INTRODUCTION}

La race d'abeilles de la Péninsule Ibérique (Apis mellifera iberica) appartenant au groupe des populations du Nord-Ouest de l'Europe (Du Praw, 1965 a ; ROTHENBUHLER et al., 1968) représente un tronc d'évolution morphogénétique très différent de celui des populations du Sud-Est de l'Europe (Rutrner, 1973 ; RUTTNER et al., 1978).

Afin de connaître les écotypes éventuels des abeilles espagnoles, nous avons étudié des populations provenant de différentes régions géographiques situées des deux côtés de la Cordillère Cantabrique ainsi que plusieurs autres situées sur la Cordillère elle-même. Ces zones ont été choisies parce que tout en étant limitrophes, elles offrent un contraste climatologique très net, et la Cordillère ellemême, avec une altitude de 1500 mètres, pourrait agir comme barrière géographique empêchant la migration entre les deux zones. La région du Nord, limitrophe avec la mer Cantabrique, possède un climat maritime avec un index de pluviosité élevé. La région située au Sud de la Cordillère est un plateau avec une altitude moyenne de 700 mètres qui possède un climat caractéristique du genre continental avec une grande variation thermique entre l'hiver et l'été (FonT, 1983). En conséquence, la flore mellifère est nettement différente. 
Cette étude a été réalisée à l'aide d'une analyse multivariate sur quelques caractères morphologiques. Ce genre d'analyse a déjà été utilisé par d'autres auteurs avec des bons résultats, permettant la séparation d'entités infraspécifiques chez l'abeille (Du Praw, 1964, 1965 a ; Tomassone et Fresnaye, 1971 ; CoRnuet et al., 1975).

\section{MATÉRIEL ET METHODES}

Les échantillons ont été recueillis au cours des mois de juillet et d'août 1984 dans les deux régions indiquées et dans la région montagneuse intermédiaire définie par des altitudes supérieures à 1000 mètres. Sur la figure 1 on peut voir la localisation des 53 points d'échantillonnage, choisis en essayant d'obtenir une distribution uniforme sur le territoire. Avec ceci, nous essayerons de constater s'il y a des groupements que l'on pourrait, a posteriori, mettre en rapport avec la situation géographique des échantillons. Nous avons choisi des ruches autochtones ainsi que des échantillons de deux ruches provenant du plateau et introduites dans le Nord depuis 4 et 6 années, respectivement, sans aucun contrôle des accouplements. Chaque échantillon est constitué de 20 ouvrières provenant de la même ruche. Pour réaliser cette analyse, on a choisi les 13 angles et les deux mesures de longueur et largeur de l'aile (Fig. 2) proposés par Du Praw (1964) pour la discrimination des races puisqu'ils sont facilement mesurables et ont peu de corrélation entre eux (Du Praw, 1964). On a aussi calculé l'index cubital car il ne demande pas trop de travail supplémentaire et il a déjà été employé par d'autres auteurs. Tous ces caractères se sont avérés très utiles dans la discrimination des groupes d'abeilles (Du PRAW, 1964, 1965 a et b; RUTTNER, 1973, 1976). Les mesures ont été réalisées comme suit : on découpe les ailes droites antérieures de chaque abeille ouvrière, on les met sur un verre porte-objets par groupes de cinq, et on projette chaque préparation avec un grossissement de $18 \mathrm{x}$ à l'aide d'un agrandisseur photographique sur une feuille de papier millimétré. On marque sur ce papier, avec une aiguille, les points d'intersection des veines de l'aile. A partir des coordonnées de chaque point on a calculé et enregistré les différents paramètres dans un ordinateur.

Etant donné que nous ne disposons pas de critères solides pour former des groupes, les renseignements obtenus ont été soumis à l'analyse canonique sans réaliser aucun groupement a priori des échantillons. Cette façon de réaliser l'analyse en prenant comme groupe chacun des échantillons, a été proposée par Cornuet et al. (1975) et a été utilisée par Louis \& LeFEBVRE (1971) et Cornuet et al. (1978) avec de bons résultats. L'analyse donne comme résultat une série d'équations orthogonales déterminées par les coefficients qu'il faut appliquer aux 16 variables que l'on a mesurées. Les variables qui présentent une plus grande variation parmi les échantillons par rapport à la variation dans l'échantillon, ont plus de poids dans les équations. D'autre part, on attribue aussi une plus grande importance aux variables moins corrélées avec le reste. Ces variables sont celles qui contribuent le plus à la formation des axes canoniques, parmi lesquels on en choisira, pour la représentation graphique et la définition des groupes, deux ou trois qui puissent expliquer le pourcentage le plus élevé de la variation.

En partant des résultats de l'analyse canonique on a réalisé un dendrogramme suivant la méthode UPGMA (SOKal \& Michener, 1958) dans le but d'établir une classification hiérarchique.

\section{RESULTATS}

Sur le tableau 1 on présente les moyennes des 53 populations pour chacun des caractères étudiés. L'écart-type des moyennes pour chaque caractère a été 


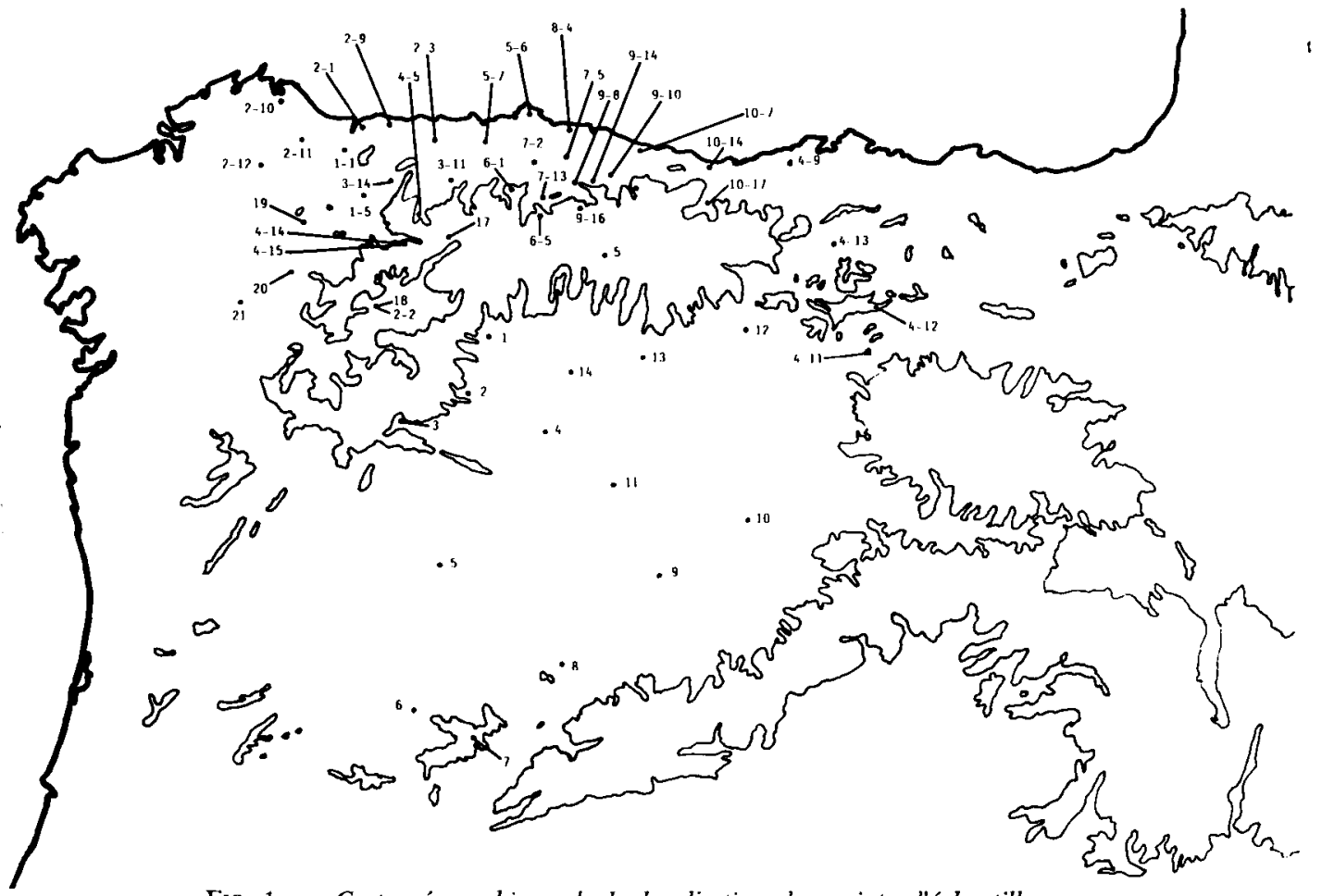

Fig. 1. - Carte géographique de la localisation des points d'échantillonnage

FIG. 1. - Geographic location of the sampling points

On montre la courbe de nivcau des $1000 \mathrm{~m}$.

The contour line for $1000 \mathrm{~m}$ is shown.

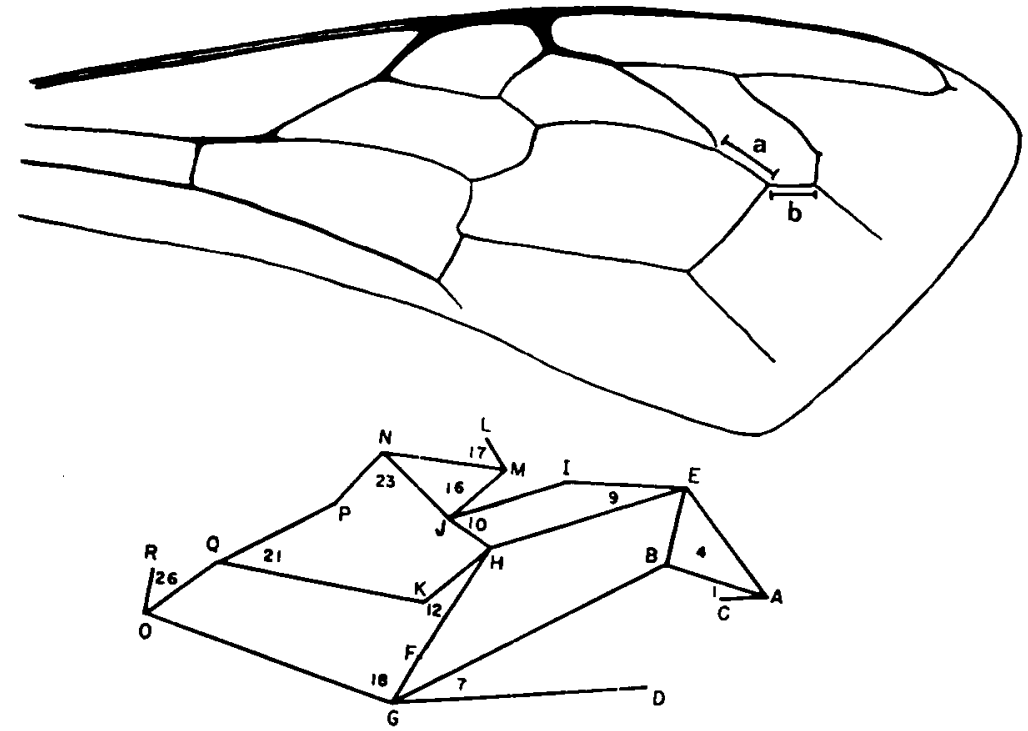

Fig. 2. - Aile antérieure d'Apis mellifera et diagramme standard de l'aile (Du Praw, 1965 a) FIG. 2. - Fore-wing of Apis mellifera and a standardized diagram of it

Caractères mesurés : index cubital $(\mathrm{a} / \mathrm{b})$; angles A-1, A-4, B-4, E-9, G-7, G-18, H-12, J-10, J-16, M-17, N-23, 0-26 et Q-21; longueur A-O et largeur L-G.

Characters measured : cubital index $(a / b)$; angles...; longer A-O and larger L-G. 


\begin{tabular}{|c|c|}
\hline$\underset{G}{\sigma}$ & 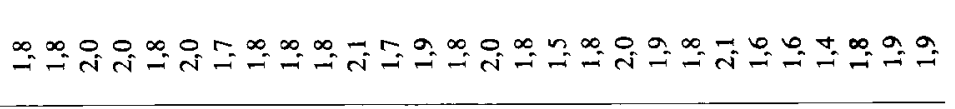 \\
\hline$\varangle$ & 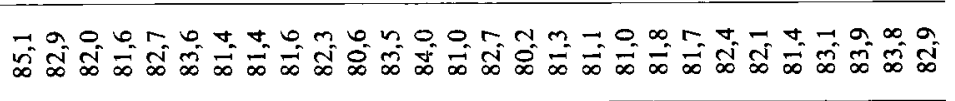 \\
\hline 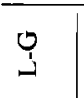 & 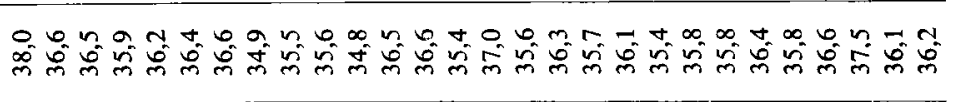 \\
\hline$\overline{\tilde{a}}$ & 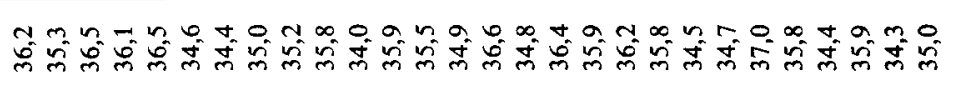 \\
\hline in & 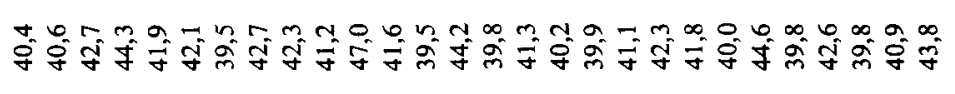 \\
\hline $\bar{z}$ & 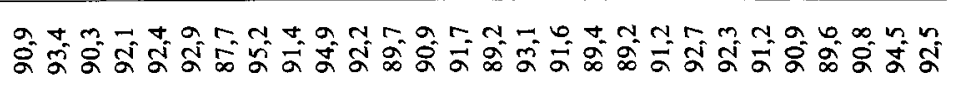 \\
\hline$\sum$ & 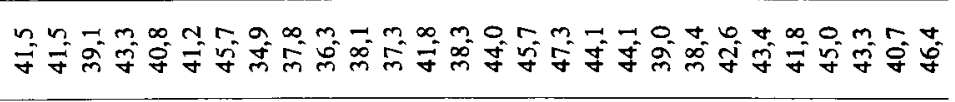 \\
\hline$\stackrel{0}{=}$ & 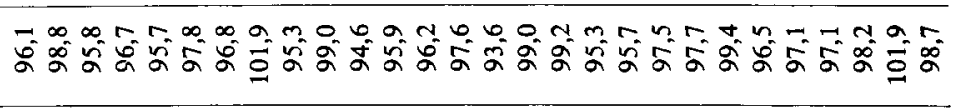 \\
\hline$\stackrel{ }{=}$ & 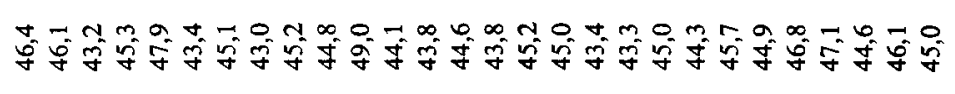 \\
\hline$\underline{I}$ & 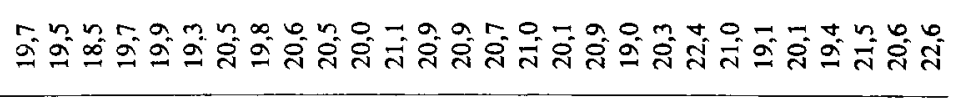 \\
\hline$\stackrel{\infty}{0}$ & 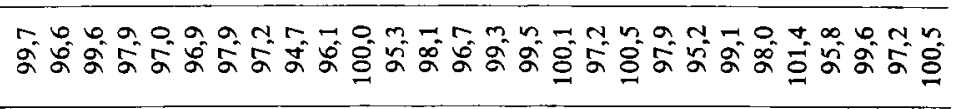 \\
\hline O & 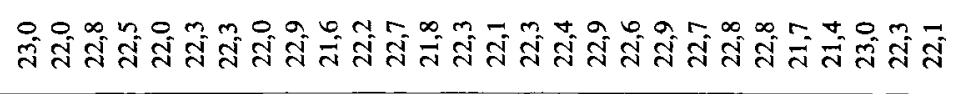 \\
\hline के & 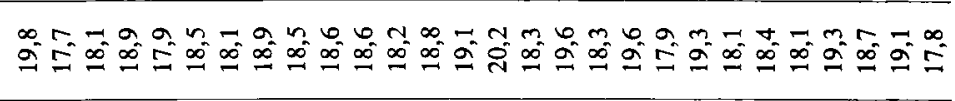 \\
\hline$\stackrel{\nabla}{\infty}$ & 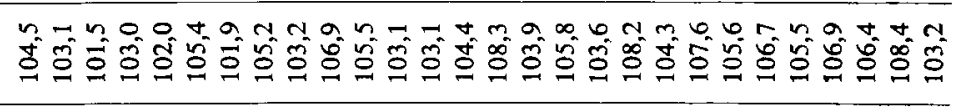 \\
\hline \& & 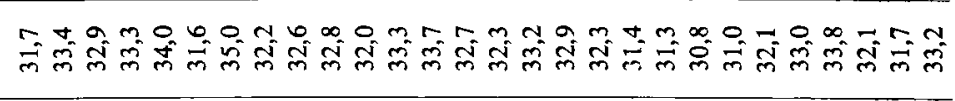 \\
\hline$\overline{4}$ & 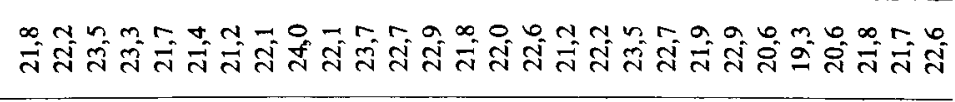 \\
\hline 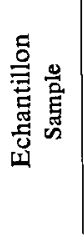 & \\
\hline
\end{tabular}




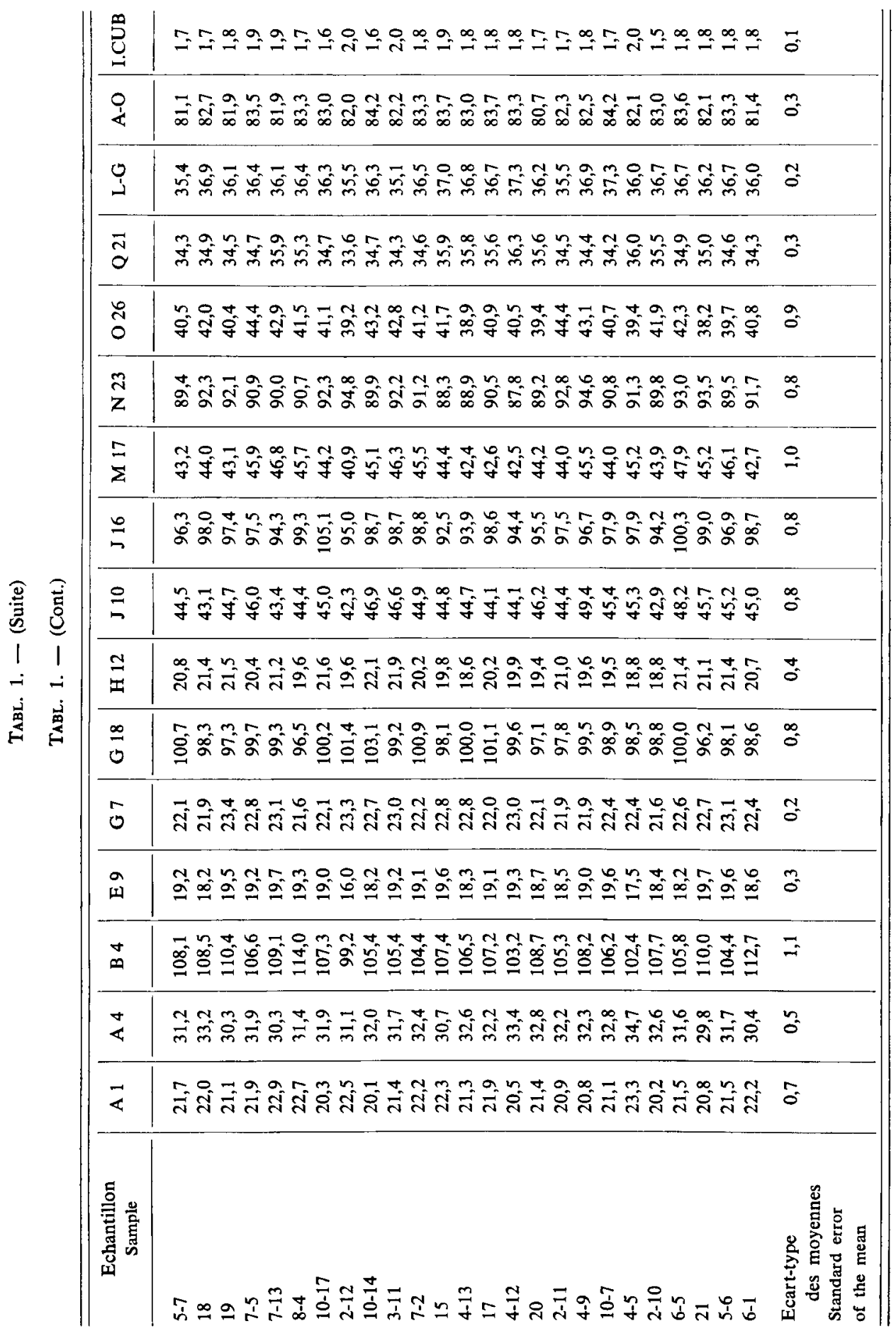


TABL. 2. - Matrice de changement des variables initiales à variables canoniques

\begin{tabular}{l|r|r|r|r|r|r|r}
\hline \multicolumn{1}{c}{$\begin{array}{c}\text { Variables } \\
\text { canoniques } \\
\text { Canonical variables }\end{array}$} & \multicolumn{1}{|c|}{ A 1 } & \multicolumn{1}{|c}{ A 4 } & B 4 & E 9 & G 7 & G 18 & \multicolumn{1}{c}{ H 12 } \\
\cline { 2 - 8 } I & $-0,012$ & 0,143 & 0,062 & 0,002 & 0,286 & 0,051 & $-0,011$ \\
II & 0,034 & $-0,007$ & $-0,047$ & 0,194 & $-0,217$ & $-0,146$ & 0,020 \\
III & 0,171 & $-0,003$ & $-0,002$ & 0,047 & 0,074 & 0,033 & $-0,006$ \\
IV & 0,012 & 0,004 & 0,018 & $-0,044$ & 0,048 & 0,011 & $-0,050$ \\
V & $-0,001$ & 0,159 & 0,037 & 0,089 & $-0,116$ & $-0,005$ & $-0,036$ \\
VI & 0,012 & 0,077 & $-0,005$ & $-0,140$ & 0,003 & $-0,063$ & 0,147 \\
VII & $-0,046$ & $-0,222$ & $-0,233$ & 0,507 & $-0,257$ & $-0,070$ & $-0,161$ \\
VIII & $-0,005$ & $-0,282$ & $-0,038$ & $-0,146$ & $-0,562$ & $-0,090$ & 0,049 \\
IX & 0,012 & 0,022 & $-0,018$ & $-0,043$ & $-0,032$ & 0,027 & 0,000 \\
X & $-0,030$ & $-0,236$ & $-0,056$ & 0,019 & $-0,455$ & 0,085 & 0,016 \\
XI & $-0,124$ & 0,156 & 0,036 & 0,177 & $-0,085$ & 0,100 & 0,139 \\
XII & $-0,003$ & 0,239 & 0,099 & 0,052 & 0,531 & 0,046 & $-0,063$ \\
XIII & 0,104 & 0,081 & 0,048 & $-0,351$ & 0,119 & 0,006 & $-0,078$ \\
XIV & $-0,015$ & $-0,419$ & $-0,078$ & 0,312 & 0,498 & $-0,099$ & 0,369 \\
XV & 0,000 & 0,312 & 0,097 & 0,128 & 0,492 & 0,083 & 0,077 \\
XVI & 0,001 & 0,192 & 0,134 & 0,233 & 0,084 & $-0,060$ & $-0,022$ \\
\hline \hline
\end{tabular}

estimé en partant du carré moyen dans un échantillon obtenu de l'ensemble des renseignements. En comparant les échantillons, nous avons obtenu une relation de WILKs égale à 0,0168 qui équivaut à une valeur $\mathrm{F}$ de 5,61 avec 832 et 15256 degrés de liberté ce qui indique que les différences entre les échantillons sont significatives à 1 pour 1000 .

Sur le tableau 2 on donne la matrice de changement des coordonnées pour obtenir les variables canoniques en fonction des variables observées. En remplaçant les moyennes de chaque échantillon dans ces formules, on peut obtenir les 16 coordonnées canoniques de chacune d'elles.

Le pourcentage de dispersion expliqué par le premier axe canonique est de $20,83 \%$. Si l'on y ajoute le second axe, on pourra expliquer les $33,35 \%$ et en ajoutant le troisième on peut atteindre $44,23 \%$ de la dispersion entre les échantillons (Tab. 3). Au moyen du test de Bartlett (1947) on constate que les 14 premiers axes sont significatifs et la dimension effective est donc 14 . Bien que la représentation canonique avec la plus grande résolution ait 14 axes, nous n'avons employé que les trois premiers. Les coordonnées canoniques de chaque échantillon sur les axes restants peuvent être obtenues en partant des formules du changement des coordonnées (Tab. 2) et des moyennes de chaque échantillon pour les variables initiales (Tab. 1).

La représentation canonique en utilisant les axes I et II (Fig. 3) montre la différence entre les échantillons du Nord et ceux du Sud de la Cordillère Cantabiique. Quoique l'on ne puisse pas établir des groupes bien définis, on peut tracer une ligne qui sépare d'un côté les échantillons provenant du Nord et d'altitudes 
TABL. 2. - Matrix of change of initial variables to canonical variables tial variables

\begin{tabular}{|c|c|c|c|c|c|c|c|c|}
\hline J 10 & J 16 & M 17 & N 23 & O 26 & Q 21 & L-G & $A-O$ & I.CUB \\
\hline 0,023 & 0,006 & 0,071 & $-0,082$ & $-0,005$ & 0,143 & 0,396 & 0,225 & $-0,807$ \\
\hline$-0,246$ & $-0,139$ & $-0,245$ & 0,146 & $-0,056$ & 0,394 & 0,617 & 0,111 & $-0,421$ \\
\hline 0,020 & $-0,020$ & $-0,004$ & 0,036 & $-0,024$ & $-0,021$ & $-0,024$ & 0,021 & $-0,979$ \\
\hline$-0,007$ & $-0,018$ & $-0,004$ & 0,008 & $-0,034$ & $-0,001$ & 0,352 & $-0,363$ & $-0,858$ \\
\hline$-0,055$ & 0,092 & 0,037 & $-0,030$ & $-0,010$ & 0,149 & $-0,191$ & 0,066 & 0,935 \\
\hline 0,041 & 0,022 & 0,023 & $-0,043$ & $-0,018$ & 0,204 & $-0,332$ & 0,121 & $-0,882$ \\
\hline$-0,043$ & $\longrightarrow 0,018$ & 0,033 & $-0,038$ & $-0,021$ & $-0,293$ & $\longrightarrow 0,132$ & 0,011 & $-0,658$ \\
\hline 0,061 & 0,168 & $-0,012$ & $-0,249$ & 0,095 & 0,110 & 0,336 & $-0,107$ & 0,576 \\
\hline 0,028 & $-0,047$ & 0,034 & $-0,057$ & 0,037 & 0,226 & $-0,261$ & 0,019 & 0,931 \\
\hline$-0,058$ & $-0,002$ & 0,006 & 0,028 & 0,034 & 0,238 & $-0,138$ & 0,044 & $-0,802$ \\
\hline 0,083 & 0,085 & $-0,105$ & $-0,151$ & $-0,052$ & $-0,002$ & $-0,097$ & $-0,007$ & 0,912 \\
\hline 0,004 & 0,110 & $-0,018$ & 0,002 & 0,116 & 0,257 & $-0,153$ & 0,044 & $-0,725$ \\
\hline$-0,237$ & $-0,153$ & 0,006 & $-0,129$ & $-0,061$ & $-0,631$ & $-0,377$ & 0,214 & $-0,389$ \\
\hline$-0,156$ & $-0,012$ & 0,026 & 0,036 & $-0,215$ & 0,014 & $-0,303$ & 0,089 & $-0,393$ \\
\hline$-0,015$ & 0,126 & $-0,025$ & 0,083 & 0,176 & 0,344 & 0,177 & $-0,101$ & $-0,638$ \\
\hline$-0,047$ & $-0,123$ & 0,003 & 0,077 & 0,022 & $-0,099$ & 0,053 & $-0,285$ & $-0,873$ \\
\hline
\end{tabular}

supérieures à 1000 mètres et de l'autre ceux du Sud. Les deux échantillons originaires du Plateau et introduits dans le Nord se situent dans le groupe des abeilles du Nord.

TABL. 3. - Test de Bartlett de la dimensionalité

et pourcentage de la variation expliquée par les «d» premières axes canoniques $(* * * ; \mathrm{p} \leq 0,001)$

TABL. 3. - Bartlett's test for the dimension and percentage of variation explained by the first « $d »$ canonical axes

\begin{tabular}{|c|c|c|c|c|}
\hline Dimension (d) & $x^{2}$ & $\begin{array}{l}\text { D.L. } \\
\text { d.f. }\end{array}$ & $\begin{array}{c}\text { Signification } \\
\text { Signifiance }\end{array}$ & $\begin{array}{c}\% \text { acumulé } \\
\text { Cumulative \% }\end{array}$ \\
\hline 0 & 4185,71 & 832 & $* * *$ & \\
\hline 1 & 3455,38 & 765 & $* * *$ & 20,83 \\
\hline 2 & 2954,74 & 700 & $* * * *$ & 33,45 \\
\hline 3 & 2513,75 & 637 & $* * *$ & 44,23 \\
\hline 4 & 2123,21 & 576 & $* * *$ & 53,47 \\
\hline 5 & 1768,93 & 517 & $* * *$ & 61,57 \\
\hline 6 & 1477,83 & 460 & $* * *$ & 68,16 \\
\hline 7 & 1207,58 & 405 & $* * *$ & 74,20 \\
\hline 8 & 982,88 & 352 & $* * * *$ & 79,12 \\
\hline 9 & 790,64 & 301 & $* * *$ & 83,25 \\
\hline 10 & 617,99 & 252 & $* * *$ & 86,93 \\
\hline 11 & 460,85 & 205 & $* * *$ & 90,25 \\
\hline 12 & 325,26 & 160 & $* * *$ & 93,20 \\
\hline 13 & 197,91 & 117 & $* * *$ & 95,91 \\
\hline 14 & 93,90 & 76 & N.S. & 98,06 \\
\hline 15 & 35,06 & 37 & N.S. & 99,24 \\
\hline
\end{tabular}




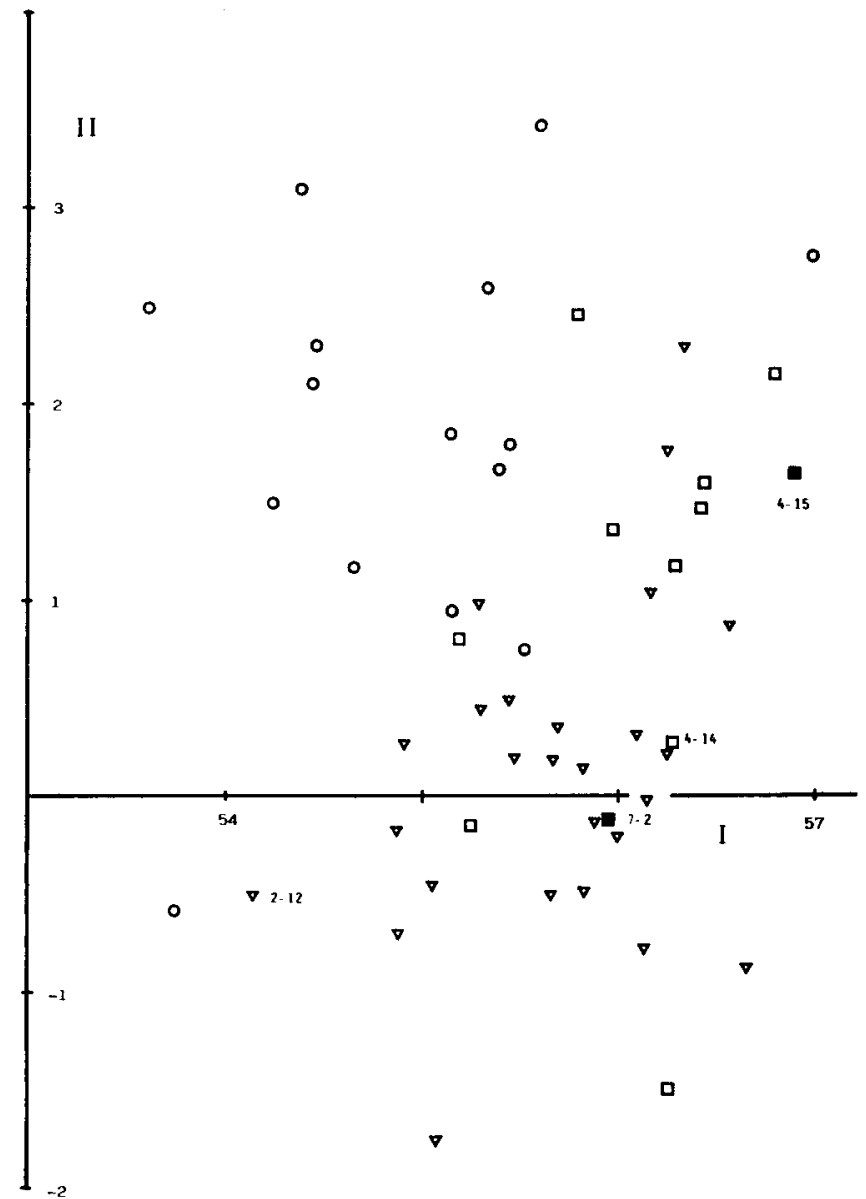

Fig. 3. - Représentation canonique sur le plan déterminé par les axes I et II

Fig. 3. - Canonical representation of the plane determined by axes I and II

$\nabla$ Ruches du Nord de la Cordillère.

Hives north of the mountains.

Ruches «intermédiaires 》 (altitudes supérieures aux $1000 \mathrm{~m}$ ). Intermediate hives (altitudes higher than $1000 \mathrm{~m}$ ).

- Ruches provenant du plateau et introduites dans le Nord. Hives from the plateau and introduced in the north.

Ruches du plateau (au Sud de la Cordillère).

Hives from the plateau (south of the mountains). 
La représentation sur le plan I-III (Fig. 4) montre un écart entre les échantillons d'origine différente du point de vue géographique quoiqu'ici la différence soit moins nette que dans la représentation antérieure. Le plan II-III (Fig. 5) sépare assez bien les échantillons du Nord et du Sud de la Cordillère. Sur ce plan les échantillons d'altitude supérieure à 1000 mètres se situent de préférence entre celles du Sud.

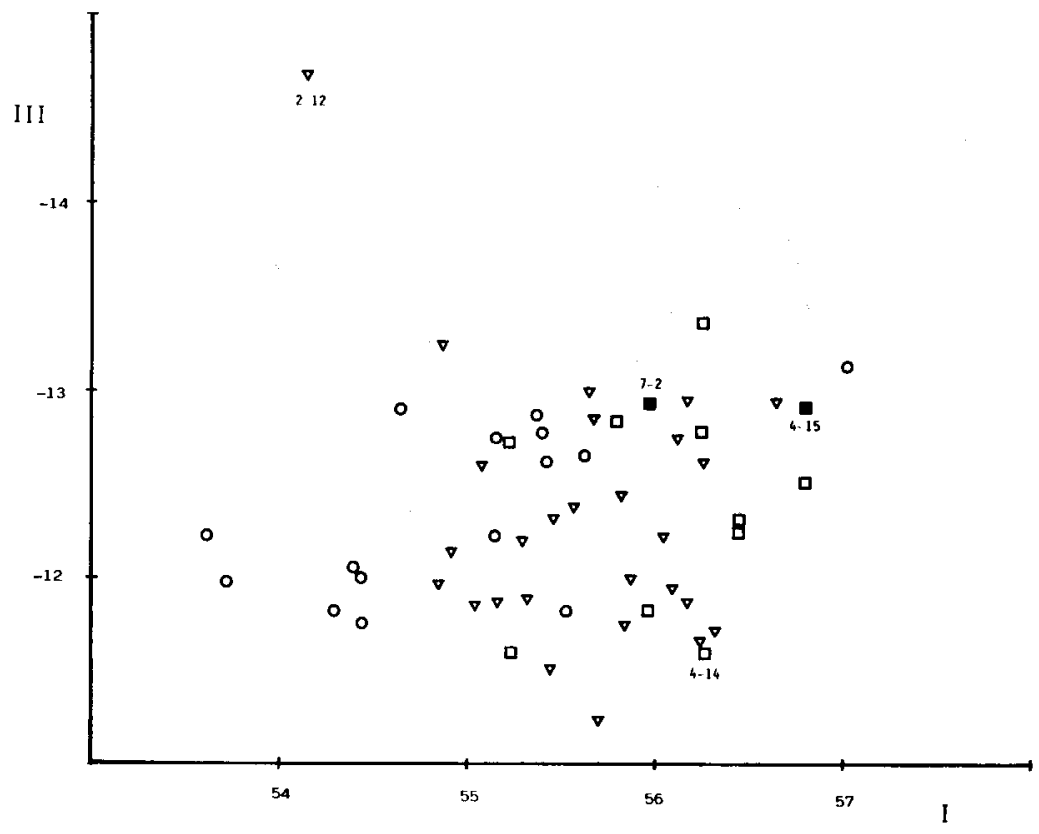

FIG. 4. - Représentation canonique sur le plan déterminé par les axes I et III

FIG. 4. - Canonical representation of the plane determined by axes I and III

Sur le dendrogramme, basé sur les distances euclidiennes entre les échantillons dans un espace déterminé par les trois premiers axes (Fig. 6), on observe deux groupes qui contiennent de préférence les échantillons provenant d'un côté ou de l'autre de la Cordillère, mais les groupes sont assez hétérogènes et de plus, la distance entre eux n'est pas importante par rapport à la distance que l'on peut observer à l'intérieur des groupes. L'échantillon 2-12 se trouve hors des deux groupes principaux.

On peut déduire, des corrélations entre les variables canoniques et les variables initiales (Tab. 4), celles qui contribuent le plus à la discrimination entre les échantillons étudiés. Dans ce cas-ci, les plus importantes seraient les mesures de la longueur et de la largeur ainsi que les angles M-17, J-16 et Q-21 puisqu'elles présentent une corrélation plus élevée avec les variables canoniques I, II et III. 


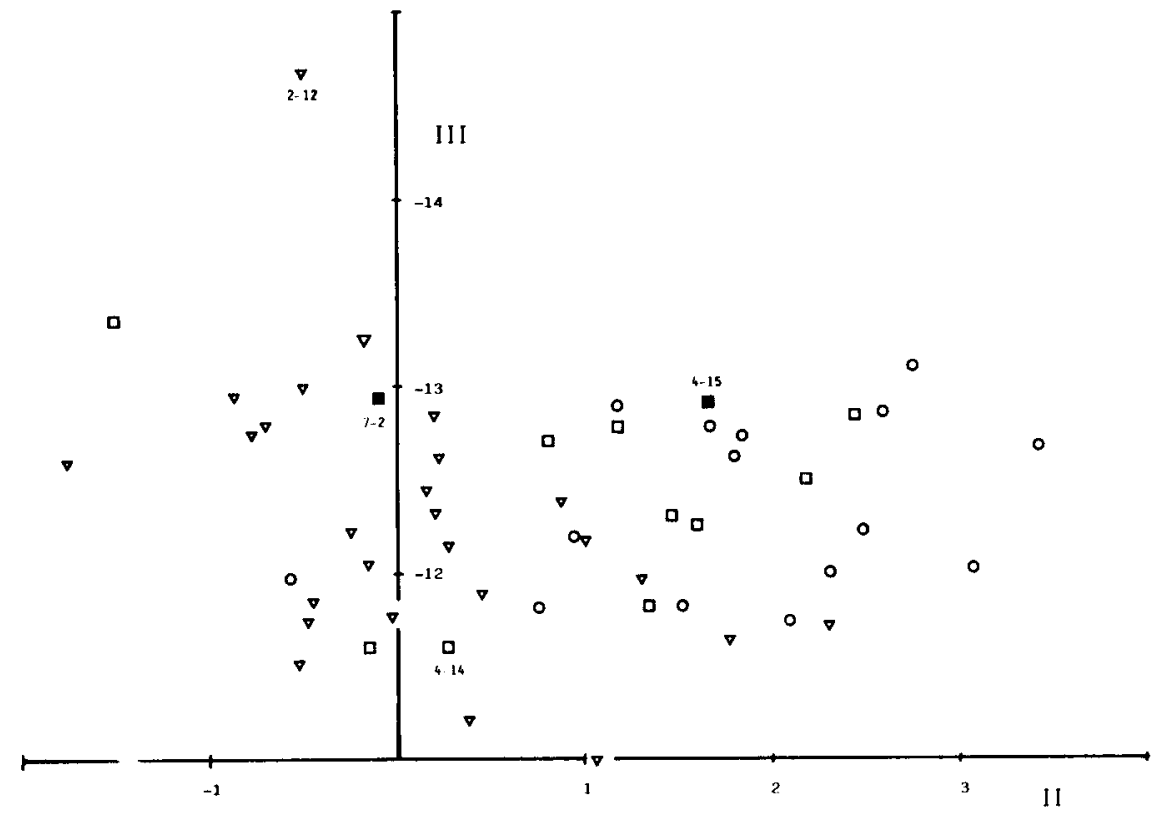

FIG. 5. - Représentation canonique sur le plan déterminé par les axes II et III FIG. 5. - Canonical representation of the plane determined by axes II and III

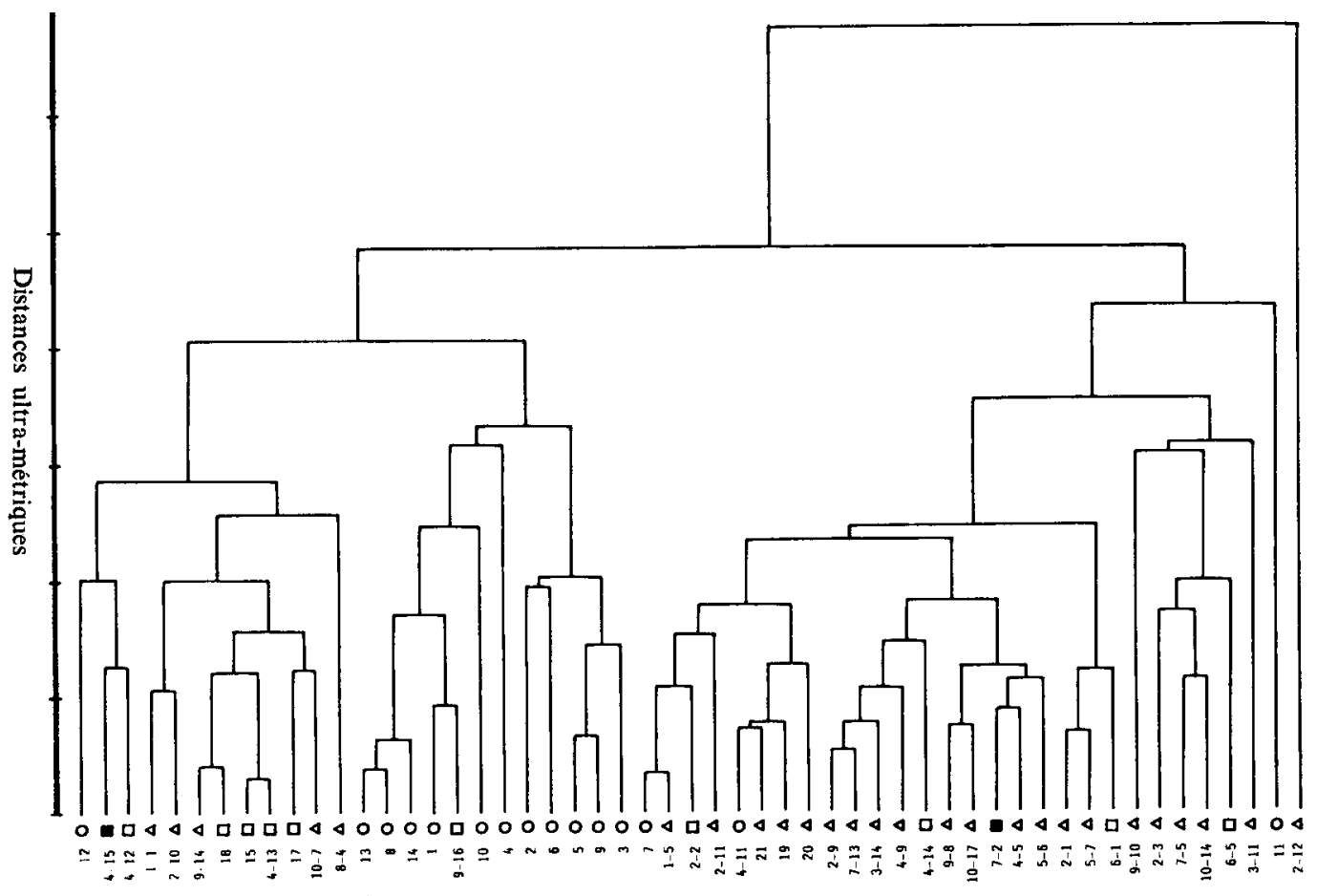

Fig. 6. - Dendrogramme basé sur les distances de Mahalanobis donnés par les axes I, II et III Fig. 6. - Dendrogram based on the Mahalanobis distances given by axes I, II and III 
TABL. 4. - Corrélation des trois premières variables canoniques avec les variables initiales

TABL. 4. - Correlation of the first three canonical variables with the initial variables

\begin{tabular}{|c|c|c|c|}
\hline $\begin{array}{l}\text { Variables initiales } \\
\text { Initial variables }\end{array}$ & I & $\begin{array}{c}\text { Canonical variables } \\
\text { Variables canoniques } \\
\text { II }\end{array}$ & III \\
\hline A 1 & $-0,096$ & 0,104 & 0,031 \\
\hline A 4 & 0,059 & 0,186 & 0,152 \\
\hline B 4 & 0,083 & $-0,121$ & 0,005 \\
\hline E 9 & 0,136 & 0,120 & 0,020 \\
\hline G 7 & 0,025 & $-0,086$ & $-0,147$ \\
\hline G 18 & 0,122 & $-0,495$ & $-0,001$ \\
\hline H 12 & $-0,039$ & $-0,276$ & $-0,078$ \\
\hline J 10 & 0,024 & $-0,376$ & $-0,194$ \\
\hline J 16 & $-0,183$ & $-0,369$ & 0,266 \\
\hline M 17 & 0,329 & $-0,894$ & 0,144 \\
\hline N 23 & $-0,224$ & $-0,091$ & 0,067 \\
\hline O 26 & $-0,102$ & $-0,121$ & $-0,017$ \\
\hline Q 21 & 0,150 & 0,623 & 0,176 \\
\hline L-G & 0,570 & 0,431 & $-0,038$ \\
\hline A-O & 0,389 & 0,343 & $-0,072$ \\
\hline I.CUB & $-0,175$ & $-0,026$ & 0,107 \\
\hline
\end{tabular}

En dernier lieu, nous avons calculé les coordonnées correspondant aux échantillons étudiés dans ce travail selon les fonctions discriminatoires de la classification 4 donnée par Du Praw (1965 a). Cette classification sert à différencier les populations d'Europe et celles d'Afrique. On trouve que les 53 échantillons étudiés appartenaient au groupe des populations du Nord et de l'Ouest de l'Europe et du Nord de l'Afrique et qu'ils sont séparés des populations de l'Afrique Centrale et du Sud et de celles du Sud-Est de l'Europe. Ceci corrobore l'utilité de ces caractères pour la taxonomie des abeilles.

\section{DISCUSSION}

Les points qui correspondent aux colonies échantillonnées se distribuent dans les plans déterminés par les trois premiers axes canoniques en formant un nuage de points avec une nette polarité, de telle façon que, même s'il n'y a aucune solution de continuité qui permette d'établir une différence entre les groupes de colonies, il est toujours possible de tracer une ligne qui sépare les colonies situées au Nord de celles qui sont situées au Sud de la Cordillère Cantabrique. Les colonies auxquelles nous avons donné le nom d' "intermédiaires», se conduisent de façon variable, en se situant plus près des colonies du Nord ou du Sud selon le plan que l'on considère. 
Sur l'ensemble des trois axes (Fig. 6), on peut établir deux groupes principaux : un groupe qui comprend la plupart des colonies du Nord, et un autre groupe nettement divisé en deux sous-groupes. Dans un de ces sous-groupes sont inclues la plupart des colonies du Sud et dans l'autre, il y a un mélange de colonies du Nord et de colonies «intermédiaires» qui ne gardent entre elles aucune relation apparente du point de vue de la situation géographique.

La colonie 2-12 apparaît nettement différente de toutes les autres. Il faut souligner le fait que les distances à l'intérieur des groupes sont assez grandes par rapport aux distances entre les groupes.

L'absence de discrimination nette (Fig. 3, 4, 5 et 6) peut être due à la façon dont l'échantillonnage a été réalisé, puisque nous avons essayé de couvrir, de la façon la plus uniforme possible, la zone étudiée dans le but de constater s'il existait des groupes qui n'auraient pas été établis auparavant. On doit s'attendre à ce que le pouvoir de discrimination de cette analyse soit sensiblement inférieur à celui d'autres pour lesquelles l'on utilise des méthodes d'échantillonnage différentes (Louis et Lefebvre, 1971 ; Connuet et al., 1978 ; Leporati et al., 1984) parce qu'elles étaient destinées à établir les différences objectives entre les races ou les écotypes déterminés au préalable par d'autres critères. Dans ce sens, il faut souligner que nous avons trouvé une plus grande discrimination dans un travail préaiable (IzQuiERdo et al., 1985), dans lequel l'échantillonnage n'a pas été uniforme, puisque l'on a recueilli trois groupes d'échantillons, deux situés au Nord et un autre au Sud de la Cordillère.

D'autre part, il y a une donnée qui suggère que l'analyse a pris en considération certaines composantes du milieu. Les axes I et II présentent une corrélation importante et positive avec la longueur et la largeur de l'aile, c'est-à-dire qu'un des caractères qui a contribué à la différenciation des échantillons est la dimension de l'aile (Tab. 4). Ce caractère, chez Drosophila est très corrélé avec les dimensions du corps (DAvid et al., 1980), et ce dernier est très influencé par les conditions alimentaires de la larve (BAKKer, 1961; Thomas-Ourillard, 1972). Nous n'avons pas de renseignements de ce genre pour Apis mellifera, mais nous pensons que chez cette espèce, la dimension de l'aile peut présenter aussi une composante du milieu.

La conduite des colonies 7-2 et 4-15 (Fig. 3, 4, 5 et 6) ne permet pas de tirer une conclusion sur l'importance de l'effet de l'environnement parce que l'on peut supposer qu'une partie importante de leurs gènes provient de la population des lieux où elles étaient installées. Cependant, l'influence du milieu peut être peu importante parce que les colonies 4-14 et 4-15 proviennent du même rucher et sont bien différentes (Fig. 3, 4, 5 et 6).

D'après les résultats obtenus, la Cordillère Cantabrique limiterait le flux de gènes (migration dans le sens génétique) entre les populations situées de chaque côté. Elle apparaît comme le principal élément qui établit la différence entre les 
groupes (Fig. 3, 4, 5 et 6), bien qu'elle ne soit pas un accident géographique capable de produire un isolement. Ceci est en accord avec les résultats obtenus par BADino et al. (1983) qui montrent que les altitudes inférieures à $2000 \mathrm{~m}$ ne constituent pas une barrière pour les abeilles.

Reçu pour publication en aồt 1985. Accepté pour publication en décembre 1985.

\section{REMERCIEMENTS}

Les auteurs remercient l'Institut de Recherche et Développement de l'Apiculture de la Fundación Principado de Asturias pour proportionner des échantillons.

\section{SUMMARY}

BIOMETRIC ANALYSIS OF THE HONEY BEE POPULATIONS FROM THE NORTHWEST OF THE IBERIAN PENINSULA

A biometrical analysis of the honey bees from the northwest of the Iberian Peninsula was performed in order to see whether or not it is possible to define different ecotypes. The region studied includes two geographic areas which are separated by the Cantabrian chain of mountains and have very different climates.

Fifty three samples, each consisting of 20 worker bees from the same hive, were taken randomly over the region (Fig. 1). The morphological traits used to perform the multivariate analysis were the 13 angles and 2 distances of the forewing proposed by DU PRAW (1965 a) as well as the cubital index (Fig. 2). These 16 parameters were used in a canonical analysis without grouping the samples a priori.

Canonical representations using axes I, II and III (Fig. 3, 4 and 5) account for 44,23\% of the variability, and show two points corresponding to the two geographical areas located to the North and the South of the Cantabrian mountains. Nevertheless the separation is no completely clear, and from the dendrogram (Fig. 6) it can be seen that the within-group distances are fairly great compared to the distance between the two main groups.

Therefore we must conclude that the Cantabrian mountains limit the migration between the two geographical areas but this limitation is not so strong as to produce a complete isolation.

\section{ZUSAMMENFASSUNG}

\section{BIOMETRISCHE UNTERSUCHUNG VON BIENENPOPULATIONEN (APIS MELLIFERA) AUS NORDWEST-SPANIEN}

Es wurde eine biometrische Analyse der Honigbienen aus dem Nordwesten der iberischen Halbinsel durchgeführt, um festzustellen, ob sich verschiedene ökotypen finden lassen. Die untersuchte Region umfaßt zwei geographische Gebiete, die durch die cantabrischen Cordilleren getrennt sind und die sehr unterschiedliches Klima haben.

Dreiundfünfzig Proben, jede aus 20 Arbeitsbienen aus demselben Volk bestehend, wurden entnommen, zufällig verteilt über die Region (Fig. 1). Die morphologischen Merkmale, die zur Multivariat-Analyse benutzt wurden, waren die 13 Winkel und 2 Distanzen des Vorderflügels, wie sie von Dupraw (1965 a) angegeben wurden, sowie der Cubitalindex (Fig. 2). Diese 16 Parameter wurden zu einer canonischen Analyse benutzt, ohne die Proben a priori in Gruppen zu teilen. 
Die canonische Darstellung mit Benutzung der Achsen I, II und III (Fig. 3, 4 und 5), die für 44,23\% der Variabilität verantwortlich sind, zeigt zwei Gruppen, die den zwei geographischen Gebieten nördlich und südlich der cantabrischen Bergkette entsprechen. Die Trennung ist jedoch nicht vollständig, und es ist aus dem Dendrogramm (Fig. 6) ersichtlich, daß die Distanzen innerhalb der Gruppe im Vergleich zu den Distanzen zwischen den beiden Hauptgruppen ziemlich groß sind.

Daraus müssen wir den Schluß ziehen, daß die Cantabrischen Cordilleren den Austausch zwischen den beiden geographischen Gebieten einschränken, aber daß diese Einschränkung nicht so stark ist, um eine vollständige Isolierung hervorzurufen.

\section{REFERENCES BIBLIOGRAPHIQUES}

Badino G., Celembrano G., Manino A., 1983. - Population structure and Mdh-1 locus variation in Apis mellifera ligustica. J. Hered., 74, 443-446.

BAKKER K., 1961. - An analysis of factors which determine success in competition for food among larvae of Drosophila melanogaster. Arch. Neerl. Zool., 14, 200-281.

Bartlett M.S., 1947, - Multivariate analysis, J. Roy. Stat. Soc., B 9, 176-197.

Cornuet J.M., Fresnaye J., Lavie P., 1978. - Etude biométrique de deux populations d'abeilles cévenoles. Apidologie, 9, 41-55.

Cornuet J.M., Fresnaye J., Tassencourt L., 1975. - Discrimination et classification de populations d'abeilles à partir de caractères biométriques. Apidologie, 6, 145-187.

David J.R., Cohet Y., Foulllet P., Arens M.F., 1980. — Phenotypic variability of wild collected Drosophila : an approach toward understanding selective pressures in natural populations. Egypt. J. Genet and Cytol., 9, 51-66.

Du Praw E.J., 1964. - Non Linnean taxonomy. Nature, 202, 849-852.

Du Praw E.J., 1965 a. - Non-linnean taxonomy and the systematics of honeybees. System. Zool., 14, $1-24$.

Du PraW E.J, $1965 \mathrm{~b}$. - The recognition and handling of honeybee specimens in non-linnean taxonomy. J. Apic. Res., 4, 71-84.

Font I., 1983. - Climatología de España y Portugal. Inst. Nac. de Metereología. Madrid.

Izquierdo J.I., Dominguez A., Albornoz J., Santiago E., 1985. - Discriminación entre poblaciones de abejas (Apis mellifera) de Asturias y la Submeseta Norte. Bol. Cien. Nat. I.D.E.A., 35, 87-101.

Leporati M., Valli M., Cavicchi S., 1984. - Etude biométrique de la variabilité géographique des populations d'Apis mellifera en Italie septentrionale. Apidologie, 15, 285-302.

Louis J., Lefebvre J., 1971. - Les races d'abeilles (Apis mellifica L.) I. Détermination par l'analyse canonique. Biométrique-Praximétrie, XII, 1-41.

Rothenbuhler W.C., Kulincevic J.M., Kerr W.C., 1968. - Bee genetics. Annu. Rev. Genet, 2, 413-438.

Ruttner F., 1973. - Die Bienenrassen des mediterranen Beckens. Apidologie, 4, 171-172.

Ruttner F., 1976. - Isolated populations of honeybees in Australia. J. Apic. Res., 15, 97-106.

Ruttner F., Tassencourt L., Louveaux J., 1978. - Biometrical-statistical analysis of the Geographic variability of Apis mellifera L. Apidologie, 9, 363-381.

Sokal R.R., Michener C.D., 1958. - A statistical method for evaluating systematic relationships. Univ. Kansas Sci. Bull, 38, 1409-1438.

Thomas-Ourillard M., 1972. - Influence de l'alimentation sur le nombre de tubes ovariens chez la Drosophile. Ann. Génét., 15, 177-182.

Tomassone R., Fresnaye J., 1971. - Etude d'une méthode biométrique et stastistique permettant la discrimination et la classification de populations d'abeilles (Apis mellifica L.). Apidologie. 2, 49-65. 